\title{
CLINICAL MANAGEMENT OF MAXILLARY OSTEONECROSIS ASSOCIATED WITH ANTIRESORPTIVE MEDICATION (MRONJ): PRESENTATION OF CLINICAL CASES
}

\author{
Rey, Eduardo A, ${ }^{1}$, Rodríguez Genta, Sergio A ${ }^{2}$, Picardo, Silvana N. ${ }^{3}$ *
}

\begin{abstract}
Author information: ${ }^{1}$ President of the National Academy of Dentistry; Consultant to the National Academy of Medicine; Former Professor of Oral and Maxillofacial Surgery I and II School of Dentistry University of Buenos Aires, ${ }^{2}$ Head of Practical Works Chair in Oral and Maxillofacial Surgery II School of Dentistry University of Buenos Aires, ${ }^{3}$ Head of Practical Works Chair in Oral and Maxillofacial Surgery II School of Dentistry University of Buenos Aires and Department of Dentistry Favaloro Foundation University Hospital.
\end{abstract}

\begin{abstract}
Antiresorptive drugs: Bisphosphonates (BPs) and Monoclonal Antibodies: Denosumab (DS) are known to suppress osteoclastic activity, affecting the expression of the RANKL (Kappa $\beta$ Nuclear Activation Receptor), which corresponds to an osteoblastic differentiation factor and which is secreted by said cells, being responsible for inducing reabsorption by osteoclasts. Under certain circumstances, those medications may induce the development of Maxillary Osteonecrosis (MRONJ).

The paper is aimed to share our experience of MRONJ treatment using minimally invasive therapies (including washes and antibiotics) that does not expand the necrotic bed volumetrically and provide non-recurrent resolution of the lesion. The patients we described were on long-term therapy either with BPs or DS.

Conclusion: Interaction between health professional is essential for MRONJ prevention. The therapeutics consolidated in non-invasive maneuvers, and the manipulation of bone tissue with close follow up allows to avoid spread to deep planes. The pathological process could be successfully treated, and it is not necessary to suspend antiresorptive medications.
\end{abstract}

Keywords: Bisphosphonates (BPs), Denosumab (DS), Medication-Related Osteonecrosis of the Jaw (MRONJ)

INTRODUCTION Although Maxillary Osteonecrosis (MRONJ) is a sequel to the treatment of the last generation BPs administered intravenously, MRONJ induced by orally supplied BPs exists, although it is less frequent, like those developed by DS. For this reason, preventive measures should be implemented, including dental consultation before starting antiresorptive drug therapy and post-surgical control of oral interventions involving bone tissue. It is especially important if there is torpid bone scarring in the interval where the patient is under treatment with BPs or DS with the diagnosis of MRONJ. The goal is to avoid bone manipulation to prevent progression to clinical stages of MRONJ with greater morbidity and prevent unfavorable evolutions [1].

\footnotetext{
*Corresponding author: Ph.D. DDS. Picardo, Silvana N. Head of Practical Works Chair in Oral and Maxillofacial Surgery II School of the Dentistry University of Buenos Aires and Department of Dentistry Favaloro Foundation University Hospital.

E-mail: silvana_picardo@hotmail.com
}

The American Surgery of Bone Mineral Research (ASBMR) in 2007 defined MRONJ as "necrotic bone area exposed to the oral environment with more than eight weeks of permanence, in the presence of chronic treatment with BPs, in the absence of radiation therapy to the head and neck." In 2014 the American Association of Oral and Maxillofacial Surgeons (AAOMS) divided the MRONJ into four stages from 0 to 3 , according to the clinical and radiological aspect of the osteonecrotic lesion: stage 0 : Osteonecrotic lesion without sign-pathognomonic evidence of osteonecrosis: stage 1: osteonecrotic lesion with clinical signs and absence of clinical symptoms; Stage 2: Osteonecrotic lesion with the sign and evident clinical symptoms; Stage 3: Osteonecrotic lesion with signs and evident symptoms that involve noble structures: pathological fractures, anesthesia of the lower dental nerve, oral-nasal communication, oral-sinus communication, skin fistulas [2].

It should be noted that these MRONJ bone lesions only have competence in the maxillary bones, a situation that follows from their ectomesenchymal origin in the presence of teeth that are involved in masticatory function (tolerating occlusal forces) and maintaining a 
dynamic balance with the indigenous flora of the over contaminated oral cavity in patients with the pre-existing infectious disease [3].

It is known that MRONJ presents a distinctive anatomopathological pattern that characterizes them, both in prescribed patients with BPs or with DS: "Pagetoid bone-like histological pattern, with signs of mosaic remodeling and trabecular appearance, areas of necrosis and bacterial colonies in surface and between medullary spaces" [4].

It is also known that not all patients under treatment with antiresorptive drugs trigger MRONJ symptoms, but that there is an incidence between $0.8 \%$ and $12 \%$ in cancer patients and between 0.01 and $0.04 \%$ in osteoporotic patients [2].

In addition, the relationship of MRONJ with the genomic biomarking of certain sequences was studied, not confirming statistically significant results. In 2015, the study GENVABO (study-genetic variants as biomarkers of jaw osteonecrosis associated with bisphosphonates) was done, concluding the existence of the possibility of differential genetic biomarking in osteoclasts, describing common genomic variables present in patients with diagnosed MRONJ [5].

\section{CLINICAL CASES}

\section{Osteoporotic patient}

Female patient, 84 years old, from Formosa (north of Argentina), osteoporotic, hypertensive, and defective kidney, under treatment with Alendronate $70 \mathrm{mg} / \mathrm{w} / 12$ years. With a history of tooth extraction $44 ; 45 ; 46$, three years before the consultation. Bone curettage every six months to achieve remission of the osteonecrotic lesion. The patient presents with the anesthesia of the Lower Dental Nerve. Diagnosis of certainty: MRONJ clinical stage 3 (Figure 1).

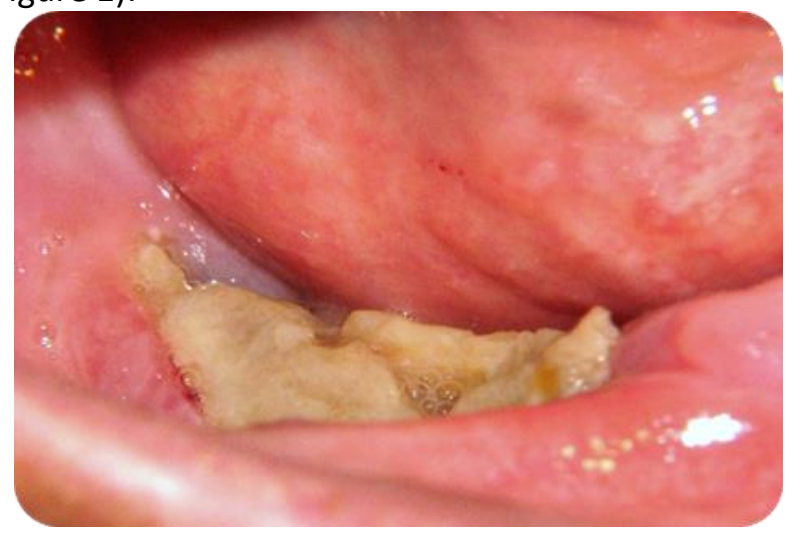

Figure 1. Diagnosis of certainty: MRONJ clinical stage 3
The patient reported that she had not used her lower complete prosthesis since MRONJ manifested herself. The clinical doctor requested an inter-consultation since she had a frank decrease in her body mass. (Figure 2 ).

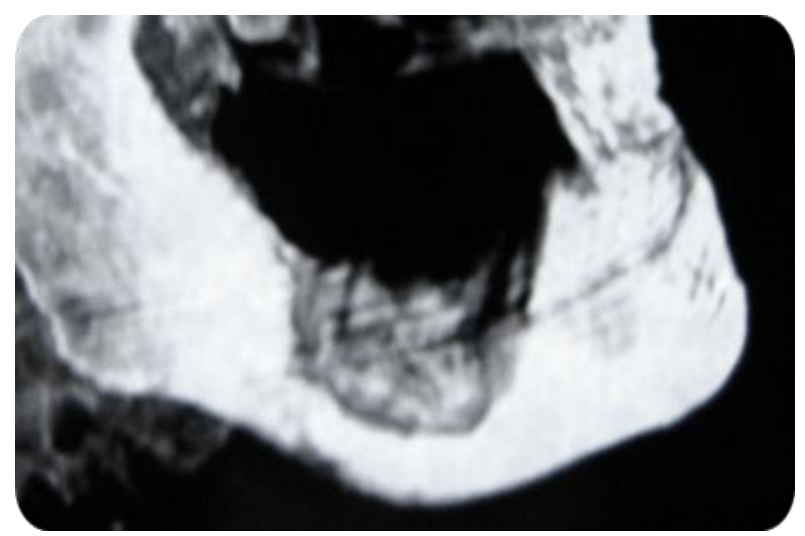

Figure 2. Computed tomography showing radiolucent lesion MRONJ.

Antiseptic washes were started with $0.12 \%$ Chlorhexidine, $10 \%$ Povidone iodine and $0.05 \%$ Rifamycin, alternating them monthly to produce the reflux of the inflammatory content, opportunely accompanied with antibiotic therapy: Amoxicillin 500 mg + clavulanic acid 125 mg every 8 hours for seven days, talking with the treating doctor, accompanying your two systemic clinical exacerbations (lymphadenopathy, fever, tumor). The clinical picture of MRONJ remitted in six months by spontaneously expelling the bone sequestration, reconfirming its diagnosis with the support of the Laboratory of Pathological Anatomy. Her soft tissues recovered without presenting evident clinical and / or radiological lesions or recurrences in fourteen years. Prosthetic rehabilitation was indicated (Figure 3).

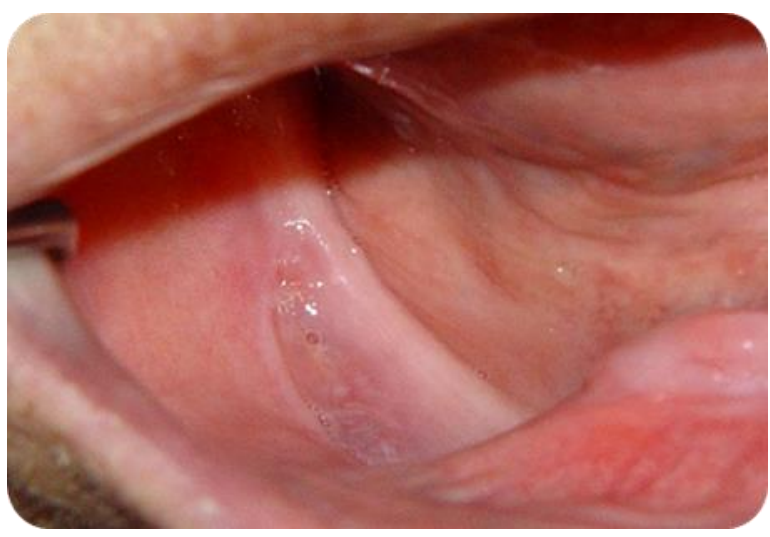

Figure 3. Soft jaw tissues recovered under atraumatic treatment MRONJ. 


\section{Oncology Patient}

Female patient, resident in CABA, 72 years, history of breast $A C$ : malignant pulmonary hypercalcemia, allergic to lodine, under treatment with DENOSUMAB $60 \mathrm{mg} / \mathrm{ml} / 2$ years, she had the previous prescription of Zoledronic acid $4 \mathrm{mg} / 3$ years.

He was presented with a panoramic X-ray showing a radiolucent image, painless, suppurating without systemic compromise with frank over contaminated bone exposure. The injury started after tooth extraction 46 with six months of evolution corresponding MRONJ stage 2, without pathological stimulation with the Lower Dental Nerve despite its proximity (Figure 4).

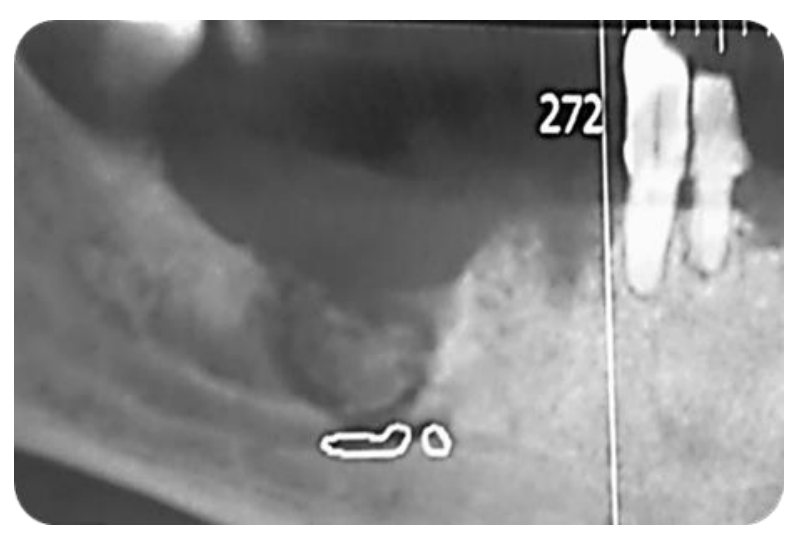

Figure 4. MRONJ stage 2 showing radiolucent sequestration over Lower Dental Nerve.

Antiseptic washes were started with $0.12 \%$ Chlorhexidine and $0.05 \%$ Rifamycin, alternating them monthly to produce the inflammatory content's reflux, opportunely accompanied by antibiotic therapy: ciprofloxacin $500 \mathrm{mg}$ every 12 hours for seven days, indicated in consultation with the oncologist. After the first exacerbation, the patient exfoliated a bone fragment sent to the Laboratory of Pathological Anatomy to reconfirm the correct diagnosis. Spontaneous total exfoliation of the MRONJ lesion was achieved in four years, under antiseptic treatment and timely antibiotic follow-up at times of clinical exacerbation. Neuronal injury was absent and wound closure. A referral was made for her final prosthetic rehabilitation since, during the expulsion of the necrosis, the patient used her current prosthesis relieved at the MRONJ lesion location (Figure 5).

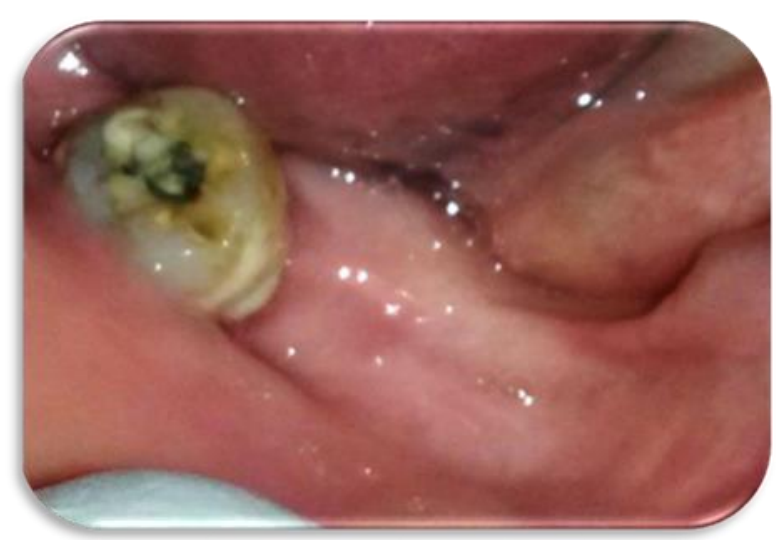

Figure 5. Clinical soft jaw tissues recovered under atraumatic treatment MRONJ.

After spontaneous exfoliation of MRONJ sector 4 with a 10-year follow-up in the panoramic radiograph, a maxillary bone defect and trabeculae bone were observed with no apparent inflammatory lesion compatible with the clinic. Until now, the lesion was not recurrent (Figure 6).

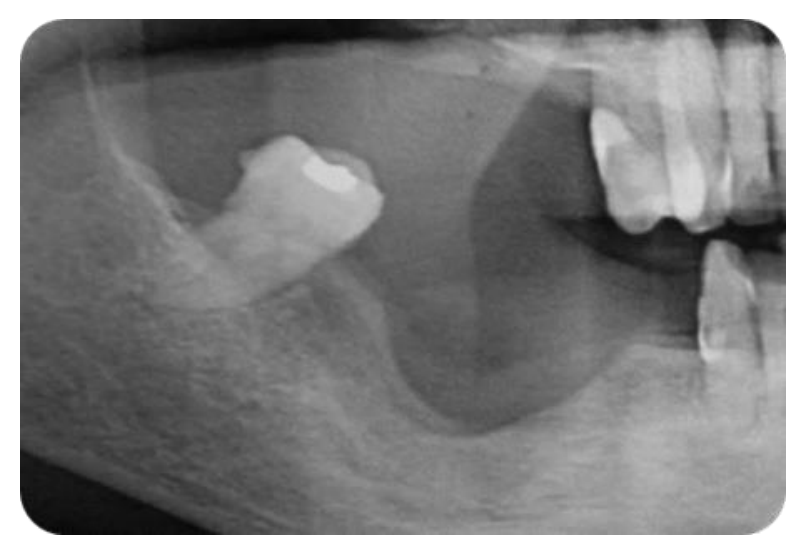

Figure 6. Radiological bone jaw tissues after under atraumatic treatment MRONJ.

DISCASSION The authors believed it is necessary to make a differential diagnosis of different infectious pathologies that involve bone tissue with respect to their etiology. From the radiological clinical point of view, the pathologies described below have similar characteristics [6]. It is known that, in osteomyelitis, that the cause is microbiological; therefore: with the culture the Antibiograms emerge, performing the correct resection maneuver, the evolution is favorable since the osteoblastosteoclast cell coupling is not involved in bone healing [2]. 
In the case of osteoradionecrosis, the etiological factor is physical, which causes damage to the cellular genetic material, both of the osteoblast and the osteoclast temporarily according to the potential of radiation, but, even so, when the time elapses, the resolution therapeutic is identical to the treatment of osteomyelitis [7].

In the case of MRONJ, its fundamental characteristic is positioned in the biochemical particularity of the pharmacokinetic expression of antiresorptive drugs, reversibly (DS) or irreversibly (BPs) inhibiting the functionality of the osteoclast. Therefore, the authors are recommending the consideration of invading bone tissue as little as possible and performing resective therapies in cases of systemic infectious spread follows, since its longterm resolution would not be effective because the drug (BPs) has frank accumulation at a distance, a characteristic used by treating doctors; and it would not have clinical relevance to suggest its suspension [3].

If there were evaluated the reversible mechanism of action of DS in the FREEDOM study [8], the dental approach would be advisable due to its reversibility. Still, we must bear in mind the osteopathic problems associated with the abrupt suspension of treatments with this medication: spontaneous bone fractures, abrupt loss of bone mass, etc., referred to in Post Hoc FREEDOM [9]. From this point of view, it would not be recommended to request the suspension of DS under any circumstances if a doctor requires the use of the treatment. Even less in cancer patients depending on antiresorptive to avoid spreading their bone metastases [10].

Analyzing Limones A. et al.'s meta-analysis, no significant clinical differences were found concerning bone necrosis associated with BPs versus DS, coinciding with our experience [11].

Given those above, both osteoporotic and oncological pathologies require the accumulation of the drug due to the need to use antiresorptive protocols that present higher relative potencies, such as the case of treatment in oncological patients [10].

According to the recommendations of AAOMS [2], Task Force [12] and AOCMF [3] coincide with the sharing of consensus on minimally invasive manipulations once the necrotic foci have been installed and the preventive attitude prevails of eliminating all septic foci prophylactically before starting therapy with antiresorptive drugs. There are positions with a trend more committed to direct bone manipulation to evacuate the infectious problem [11] and other more conservative positions to not expand drug necrosis volumetrically due to bone accumulation of BPs or DS [3].

Given those above, both osteoporotic and oncological pathologies require drug accumulation due to the need to use antiresorptive protocols that present higher relative potencies, such as the case of treatment in cancer patients [10].

Some authors have seen some reversibility benefit of MRONJ in the administration of Teriparatide $20 \mathrm{mg} /$ day in osteoporotic patients taking into account the prescription of treatments no longer than six months due to the appearance of sarcomas [13]; The use of PRP and / or the application of laser has not shown any benefit. Access to the hyperbaric chamber only affects antiangiogenic recovery, but therapeutic success has not been proven in patients with MRONJ. There are not enough studies to support such therapies [1-14].

\section{Conclusion}

From the results found and from those published in the bibliography, it appears that the interaction between health professionals is essential since the prevention of MRONJ is better than the treatment. In this sense, prior dental evaluation of patients is recommended to carry out dental interventions before establishing chronic treatment with antiresorptive drugs.

We suggested that treatment must be based on the therapeutic consolidated in non-invasive maneuvers regarding the manipulation of bone tissue, performing the appropriate clinical controls to avoid systemic spread to deep planes, due to its pharmacokinetics of bone accumulation that could condition a septicemia picture in affected patients, interacting with the attending physician in the event of a certain event of exacerbation of injuries that affect the patient's general health.

According to medical criteria, it is not necessary to suspend antiresorptive medication to compensate for the morbidity of the pathology, both in osteoporotic and oncological patients. It is known that in cancer patients, the suspension of antiresorptive would instigate metastatic spread and could cause a pathological fracture.

\section{REFERENCES:}

1. Picardo SN, Rey EA. Clinical Healthcare Protocol for Bisphosphonate Related Osteonecrosis of The Jaw. International Journal of Dentistry and Oral Health 2017; 3: 42-44. 
2. Khan AA, Morrison A, Hanley DA, Felsenberg D, McCauley LK et al. Diagnosis and management of osteonecrosis of the jaw: a systematic review and international consensus. J Bone Miner Res 2015; 30(1): 3-23. doi: 10.1002/jbmr.2405.

3. Fleisher KE, Kontio R, Otto S Antiresortive drug-related ostenecrosis of the jaw (ARONJ), a guide to research. AOCMF; Switzerland; 2016.

4. Paparella ML, Brandizzi D, Santini Araujo E, Cabrini RL. Osteonecrosis of the Jaw Associated with Bosphosphonates. A. Histopathological Study of 24 Cases. JMS Dent 2014; 2(3): 1037

5. Fung PL. The GENVABO study-genetic variants as biomarkers of jaw osteonecrosis associated with bisphosphonates: a large, multicenter genome-wide association study and detailed analyses of clinical phenotype. Doctoral Thesis: Institute, University College London, 2015.

6. Picardo SN, Rey E. Osteonecrosis of The Jaw in Patients Undergoing Long-term Treatment with Bisphosphonates: Incidence and Associated Characteristics. Journal of Clinical and Medical Case Study 2017; 2;48-54.

7. Dhanda J, Pasquier D, Newman L, Shaw R. Current Concepts in Osteoradionecrosis after Head and Neck Radiotherapy. Clin Oncol (R Coll Radiol) 2016; 28(7): 459-66. doi: 10.6696/IJHNS.201906_3(2).0003.

8. Bone HG, Wagman RB, Brandi ML, Brown JP, et al. 10 years of denosumab treatment in postmenopausal women with osteoporosis: results from the face 3 randomized FREEDOM trial and open-label extension. Lancet Diabetes Endocrinol 2017; 5 (7):513-523. doi.org/10.1016/S2213-8587(17)30138-9.
9. Cummins S R, Ferrari S, Eastell R, Gilchrist N, et al.Vertebral Fractures After Discontinuation of Denosumab: A PSot Hoc Analysis of the Randomized Placebo-Controled FREEDOM Trial and Its Extension. J Bone Miner R 2018 33(2): 190-198. doi: 10.1002/jbmr.3337.

10.Marx RE, Cillo JE Jr, Ulloa JJ. Oral bisphosphonateinduced osteonecrosis: risk factors, prediction of risk using serum CTX testing, prevention, and treatment. J Oral Maxillofac Surg. 2007 65(12): 2397-410. doi: 10.1016/j.joms.2007.08.003.

11.Limones A. Sáez-Alcaide LM, Díaz-Parreñp SA, Helm A, Bornstein $\mathrm{M}$, Molinero-Mouelle P. Medication-related osteonecrosis of the jaws (MRONJ) in cancer patients treated with denosumab Vs zoledronic acid: A systematic review and meta-analysis. Med Oral Patol Cir Bucal. 2020; 25(3): 326-336. doi: 10.4317/medoral.23324

12. Burr DB, ASBMR Task Force. Summary of ASBMR Task Force on ONJ. J. Musculoskelet Neuronal Interact. 2007; 7(4):354-5.

13. Kim KM, Rhee Y, Dae Kwon Y, Geo-Kwon T, Lee JK, Yoon Kim D. Medication related Osteonecrosis of the jaw: 2015 Position Statement of the Korean Society for bone and Mineral Research and the Korean Association of Maxillo Facial Surgeons; J Bone Metab. 2015; 22: 151-165

14. Ruggiero S, Saxena D, Tetradis S, Aghaloo T, loannidou E. Task Force on Design and Analysis in Oral Health Research: Medication-Related Osteonecrosis of the Jaw. JDR Clin Trans Res. 2018; 3(3): 222-225.3 